\title{
Leflunomide in the treatment of patients with early rheumatoid arthritis - results of a prospective non-interventional study
}

\author{
Herbert Kellner • Klaus Bornholdt • Gert Hein
}

Received: 4 December 2009 /Revised: 12 February 2010/Accepted: 24 February 2010/Published online: 23 May 2010

(C) The Author(s) 2010. This article is published with open access at Springerlink.com

\begin{abstract}
Leflunomide is effective and well tolerated in the treatment of rheumatoid arthritis (RA), however, data on its use in early RA are scarce. This study seeks to evaluate effectiveness and safety of leflunomide in the treatment of early RA in daily practice. This prospective, open-label, non-interventional, multi-center study was carried out over 24 weeks including adults with early RA $(\leq 1$ year since diagnosis). Leflunomide treatment was according to label instructions. Three hundred thirty-four patients were included. Disease activity score in 28 joints (DAS28) response (reduction in DAS2 8 of $>1.2$ or reduction of $>0.6$ and a DAS28 of $\leq 5.1$ ) was $71.9 \%$ at week 12 and $84.6 \%$ at week $24.25 .0 \%$ of patients achieved clinical remission (DAS28 $\leq 2.6$ ). Most frequently reported adverse drug reactions (ADR) were diarrhea $(3.0 \%)$, nausea $(2.4 \%)$, hypertension $(1.8 \%)$, and headache $(1.5 \%)$. Serious ADR were reported in four patients (1.2\%). Leflunomide showed the effectiveness which was to be expected from controlled studies without revealing any new or hitherto unknown side effects. Onset of action was quick and significant improve-
\end{abstract}

H. Kellner $(\bowtie)$

Division of Rheumatology,

Center for Inflammatory Joint Diseases,

KH Neuwittelsbach, Romanstr. 9,

80639 Munich, Germany

e-mail: hk@prof-dr-kellner.de

\section{K. Bornholdt}

Sanofi-Aventis Deutschland GmbH, Berlin,

Potsdamer Str. 8,

10785 Berlin, Germany

\section{G. Hein}

Department of Internal Medicine, Rheumatology,

Friedrich-Schiller-University,

07740 Jena, Germany

e-mail: gert.hein@med.uni-jena.de ment of disease was seen after 12 weeks of therapy and at even higher rates after 24 weeks irrespective of the use of a loading dose. Interestingly, the DAS28-remission rate achieved was similar to the rate seen with methotrexate or biologic therapy in other studies.

Keywords Daily practice · DAS28 response - Early rheumatoid arthritis $\cdot$ Leflunomide

\section{Introduction}

Rheumatoid arthritis (RA) is a chronic inflammatory systemic disease which primarily affects the joints and often leads to joint destruction resulting in functional impairment and invalidity.

Irreversible joint damage occurs early in the course of the disease and it is now widely accepted that early treatment of RA is associated with a better outcome [1-4].

It has been shown in several clinical trials that the disease-modifying anti-rheumatic drug (DMARD), leflunomide, is effective and well tolerated in the treatment of RA. Its beneficial effects showed in high American College of Rheumatology (ACR) response rates and comprised retardation of radiographic progression as well as improvement of physical function and health-related quality of life [5]. Symptoms of disease improved within a month of starting treatment and improvement was maintained in the longterm. Via its effect on T cells, leflunomide addresses several levels of the inflammatory cascade and has antiproliferative, anti-inflammatory, and anti-destructive abilities [6-8]. This special mode of action together with its rapid onset of action makes it a promising drug also in the treatment of early RA where the prevention of irreversible structural damage is an important goal. A subgroup analysis 
of two placebo-controlled studies showed similar efficacy of leflunomide in early ( $<2$ years of disease duration) and late disease ( $>2$ years of disease duration) in contrast to sulfasalazine which seems to have better efficacy in later disease stages [8]. However, data on leflunomide in the treatment of early RA are scarce.

The aim of this non-interventional study on leflunomide was to provide information on the effectiveness and tolerability of leflunomide in the treatment of early RA under routine care conditions.

\section{Materials and methods}

\section{Study design}

This prospective, open-label, non-interventional, multicenter observational study was carried out between May 2006 and September 2007 by rheumatologists throughout Germany. The study comprised for each patient a documentation period of 24 weeks including visits at Baseline, after 12 weeks (interim visit) and after 24 weeks (final visit). Due to the non-interventional study design, IRB review and patient informed consent were not necessary at the time of the study.

\section{Patient population}

Adult patients with early rheumatoid arthritis (defined by a maximum disease duration of 1 year since diagnosis) were eligible for inclusion if the investigator was convinced that they might profit from treatment with leflunomide and if they did not show any contraindications. The physician's decision for leflunomide treatment was based on the patient's condition and independent of study documentation.

\section{Treatment}

Dosing recommendations were according to the current summary of product characteristics of Arava ${ }^{\circledR}$ (loading dose: leflunomide $100 \mathrm{mg}$ per day for 3 days; maintenance dose: leflunomide 10 to $20 \mathrm{mg}$ per day).

\section{Statistical analysis}

The primary effectiveness parameter was the disease activity score 28 (DAS28) response rate after 24 weeks. The DAS28 was calculated based on the swollen joint count (SJC), tender joint count (TJC), erythrocyte sedimentation rate (ESR), and the patient's global health assessment on a visual analogue scale (VAS). DAS28 response was defined as good or moderate response according to the EULAR criteria [9].
The response rate was calculated including exact $95 \%$ confidence limits for binomial proportion using the $\mathrm{F}$ distribution method by Collet. The influence of glucocorticoids and MTX as concomitant medication on the effectiveness of therapy was evaluated by an analysis of covariance. This model included the change in DAS28 between start and final visit as dependent variable, baseline values as covariate and the use of glucocorticoids and MTX as two independent factors (no interaction was included into the model).

Secondary effectiveness parameters were the DAS28 response rate after 12 weeks, changes in DAS28, TJC, SJC, ESR, and C-reactive protein (CRP) during 24 weeks, changes in the health assessment questionnaire-disability index (HAQ-DI) [10] and changes in pain (assessed by VAS). The safety evaluation comprised changes in vital signs and laboratory parameters as well as the documentation/reporting of adverse events.

$P$ values of all statistical tests were of exploratory character. Missing values were not replaced.

\section{Results}

\section{Patient disposition}

A total of 174 centers throughout Germany contributed data of 334 patients. All of these patients had received leflunomide and were evaluated for safety. Twenty-six patients in whom RA had been diagnosed more than 1 year before enrollment were excluded from the effectiveness population which therefore comprised 308 patients.

Analysis of DAS28 response only considered patients with evaluable DAS28 score at baseline and at least one visit after baseline (response analysis population $=276$ patients).

Demographic and other baseline characteristics

In the mean $9.0 \pm 22.3$ (median: $3.0,99 \%$ percentile: 131.0 ) months had passed between the occurrence of first RA symptoms and diagnosis and $16.3 \pm 28.0$ (median: $9.0,99 \%$ percentile: 177.0) months between the occurrence of first symptoms and start of documentation. Demographic and other baseline characteristics are summarized in Table 1.

Treatment and observation period

Patients $(57.2 \%)$ received the recommended loading dose of $100 \mathrm{mg}$ leflunomide/day. The maintenance dose was $20 \mathrm{mg}$ leflunomide/day in $91.6 \%$ of patients and $10 \mathrm{mg}$ leflunomide/day in $8.4 \%$ of patients. In the majority of patients the dose remained unchanged during the observation period. Patients $(61.7 \%)$ were concomitantly treated 
Table 1 Demographic and other baseline characteristics (safety population)
Percentages are adjusted relative frequencies, i.e., missing values are not considered

$C C P$ cyclic citrullinated peptide, $D M A R D$ disease-modifying anti-rheumatic drug, $R A$ rheumatoid arthritis, $S D$ standard deviation

\begin{tabular}{lr} 
Age (years, mean \pm SD) & $55.8 \pm 13.2$ \\
Female $(n, \%)$ & $243(73.0)$ \\
Time since RA diagnosis, months, mean \pm SD (median) & $7.5 \pm 15.8(4.0)$ \\
Rheumatoid factor, n (\%) & $242(73.1)$ \\
Positive & $89(26.9)$ \\
Negative & \\
Anti-CCP $(n, \%)$ & $143(60.9)$ \\
Positive & $92(39.1)$ \\
Negative & $38(11.6)$ \\
Rheumatoid nodules $(n, \%)$ & $139(45.6)$ \\
Joint erosion $(n, \%)$ & $166(54.4)$ \\
Yes & $174(52.1)$ \\
No & $160(47.9)$ \\
DMARD-naïve $(n, \%)$ & $140(41.9)$ \\
DMARD pre-treatment $(n, \%)$ & $34(12.2)$ \\
Methotrexate pre-treatment & \\
Corticosteroid injection within the last 30 days $(n, \%)$ & \\
\hline
\end{tabular}

with corticosteroids. In $27.5 \%$ of patients additional DMARDs, most frequently methotrexate (22.2\%), were used, although not recommended.

Treatment with leflunomide was discontinued in $11.1 \%$ of patients. Reasons included adverse event (6.3\%), patient's request $(3.3 \%)$, and insufficient therapeutic effect (3.0\%).

The patients were observed for an average of $25.5 \pm 6.0$ weeks.

\section{DAS28 response}

A high response rate was achieved under leflunomide with $84.6 \%$ of patients showing good or moderate DAS28 response after 24 weeks.

A considerable rate of responders was already seen after 12 weeks where $71.9 \%$ of patients met the criteria of DAS28 response. Response at both time points was independent of the use of a loading dose (response rate no loading dose versus loading dose after 12 weeks, $68.2 \%$ versus $74.5 \%$, and after 24 weeks, $82.4 \%$ versus $86.2 \%$ ). An overview of the individual DAS28 response categories is given in Table 2 .

Analysis of the DAS28 response rate after 24 weeks differentiated by whether or not the patients had received pre-treatment with other $\operatorname{DMARD}(\mathrm{s})$ showed no influence of this factor. However, when the individual response categories were examined it became obvious that the rate of patients with good response (DAS28 $\leq 3.2$ and reduction by $>1.2$ ) was clearly greater in DMARD-naïve patients compared to those who had received DMARD pretreatment (44.5\% versus $25.0 \%$; see also Fig. 1).

Concomitant treatment with corticosteroids or MTX did not have an effect on DAS28-response $(p=0.86$ for CS and $p=0.27$ for MTX)

Table 2 DAS28 response rates after 12 and 24 weeks of leflunomide treatment (response analysis population)

\begin{tabular}{|c|c|c|c|c|}
\hline \multirow[t]{3}{*}{ DAS28 response } & \multicolumn{2}{|l|}{ Week 12} & \multicolumn{2}{|l|}{ Week 24} \\
\hline & \multicolumn{2}{|l|}{$N^{\mathrm{a}}=260$} & \multicolumn{2}{|l|}{$N^{\mathrm{a}}=254$} \\
\hline & $n(\%)$ & $95 \% \mathrm{CI}$ & $n(\%)$ & $95 \% \mathrm{CI}$ \\
\hline Good or moderate response & $187(71.9)$ & $66.0-77.3$ & $215(84.6)$ & $79.6-88.9$ \\
\hline Good response & $52(20.0)$ & $15.3-25.4$ & $92(36.2)$ & $30.3-42.5$ \\
\hline Moderate response & $135(51.9)$ & $45.7-58.1$ & $123(48.4)$ & $42.1-54.8$ \\
\hline Insufficient response & $73(28.1)$ & $22.7-34.0$ & $39(15.4)$ & $11.2-20.4$ \\
\hline
\end{tabular}

CI confidence interval, DAS disease activity score, Good response DAS28 score $\leq 3.2$ plus improvement of $>1.2$, Moderate response DAS 28 score $\leq 3.2$ plus improvement of $>0.6$ to $\leq 1.2$ or DAS 28 score $>3.2$ to $\leq 5.1$ plus improvement of $>0.6$ or DAS 28 score $>5.1$ plus improvement of $>1.2$, Insufficient response improvement in DAS28 score of $\leq 0.6$ or improvement of $>0.6$ to $\leq 1.2$ plus DAS28 score $>5.1$

${ }^{\text {a }}$ Patients with evaluable data 
Fig. 1 DAS28 response rate after 24 weeks by DMARD pre-treatment (response population). $D A S$ disease activity score

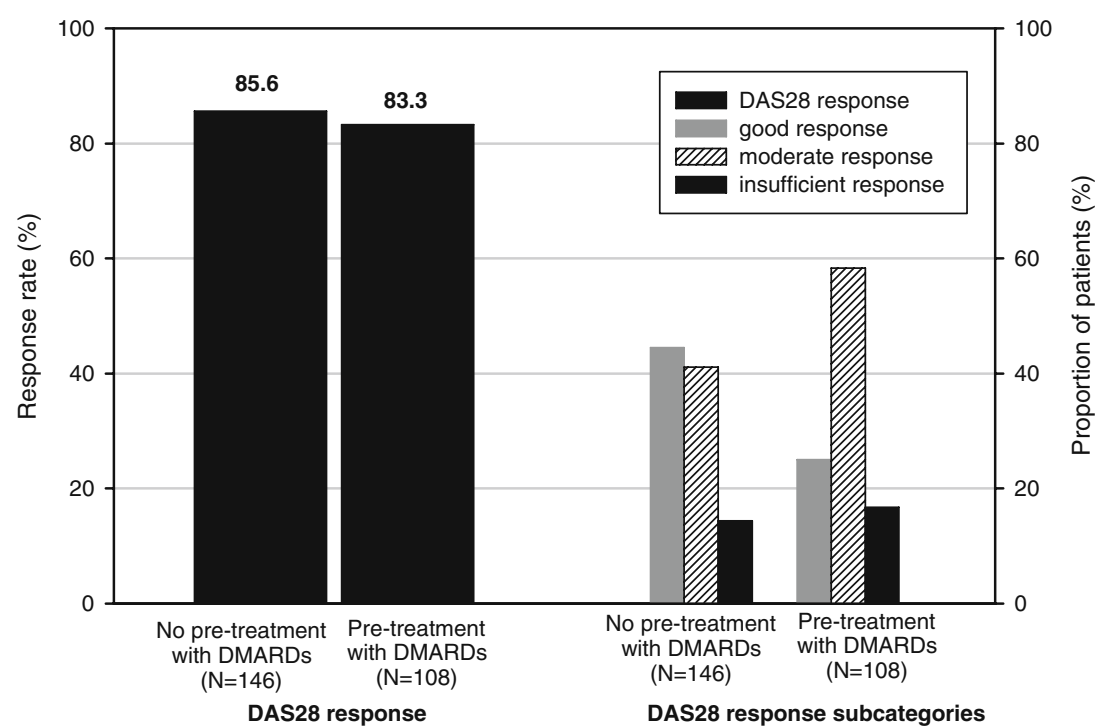

\section{Course of DAS28 score}

As illustrated by Fig. 2, disease activity improved markedly during the course of the study with the most pronounced improvement already visible after 12 weeks of treatment and a further decrease in disease activity by week 24 . The proportion of patients with high disease activity (DAS28 score $>5.1-10$ ) was clearly reduced from $71.0 \%$ at baseline to $13.8 \%$ at the final visit while the rates of clinical remission (0-2.6), low disease activity $(>2.6-3.2)$ and moderate disease activity $(>3.2-5.1)$ showed an increase. After 24 weeks of treatment $25.0 \%$ of patients were in clinical remission.

Accordingly, mean DAS28 score declined from 5.7 \pm 1.2 at baseline to $4.2 \pm 1.4$ after 12 weeks and $3.7 \pm 1.4$ at the final visit (see also Fig. 3). Endpoint analysis ${ }^{1}$ of the difference between final visit and baseline showed a mean reduction of $-2.1 \pm 1.6(p<0.001$, Wilcoxon signed rank test).

TJC, SJC, erythrocyte sedimentation rate, and CRP

The results described above for the DAS28 score were reflected in the individual components TJC, SJC, and ESR as well as in CRP (as summarized in Table 3). The objective parameters number of swollen joints, ESR, and CRP as well as the subjective component number of tender joints had markedly improved by week 12 and showed a further decrease by week 24 .

\footnotetext{
${ }^{1}$ Including only patients with data both for baseline and final visit $(N=254)$.
}

HAQ-DI and -pain scale

As shown in Table 4 the patient-reported outcome measures HAQ-DI and pain both showed considerable improvement during treatment with leflunomide which again was already observed at week 12 .

Safety

Adverse events were documented for 36 patients (10.8\%), which were considered by the investigator as being drugrelated in 32 patients $(9.6 \%)$. Most frequently reported adverse drug reactions (ADR) were diarrhea (3.0\%), nausea $(2.4 \%)$, hypertension $(1.8 \%)$, and headache $(1.5 \%)$. Serious ADR were reported in four patients $(1.2 \%)$ and comprised hypertension, pneumonia, productive cough, pyrexia, erysipelas, vomiting nausea, and hypotension. All of these patients recovered completely. Four cases of unexpected ADR were hypotension, dyspnea, dry throat, and glossodynia.

In addition to the above-mentioned adverse events, elevations in liver transaminases (as described in the summary of product characteristics) from normal to $\geq 2 \times$ upper limit of normal were noted in seven patients $(6.6 \%$; $N=106$ with normal value at baseline) for alanine aminotransferase and in two patients $(5.1 \% ; N=39$ with normal value at baseline) for aspartate aminotransferase ${ }^{2}$. Increases in transaminases from normal values to values $\geq 3 \times$ upper limit of normal were not seen.

\footnotetext{
$\overline{{ }^{2} \text { Since normal }}$ range depends on gender, one patient with missing data on gender could not be taken into account.
} 
Fig. 2 Course of DAS28 (categorical, effectiveness population). $D A S$ disease activity score

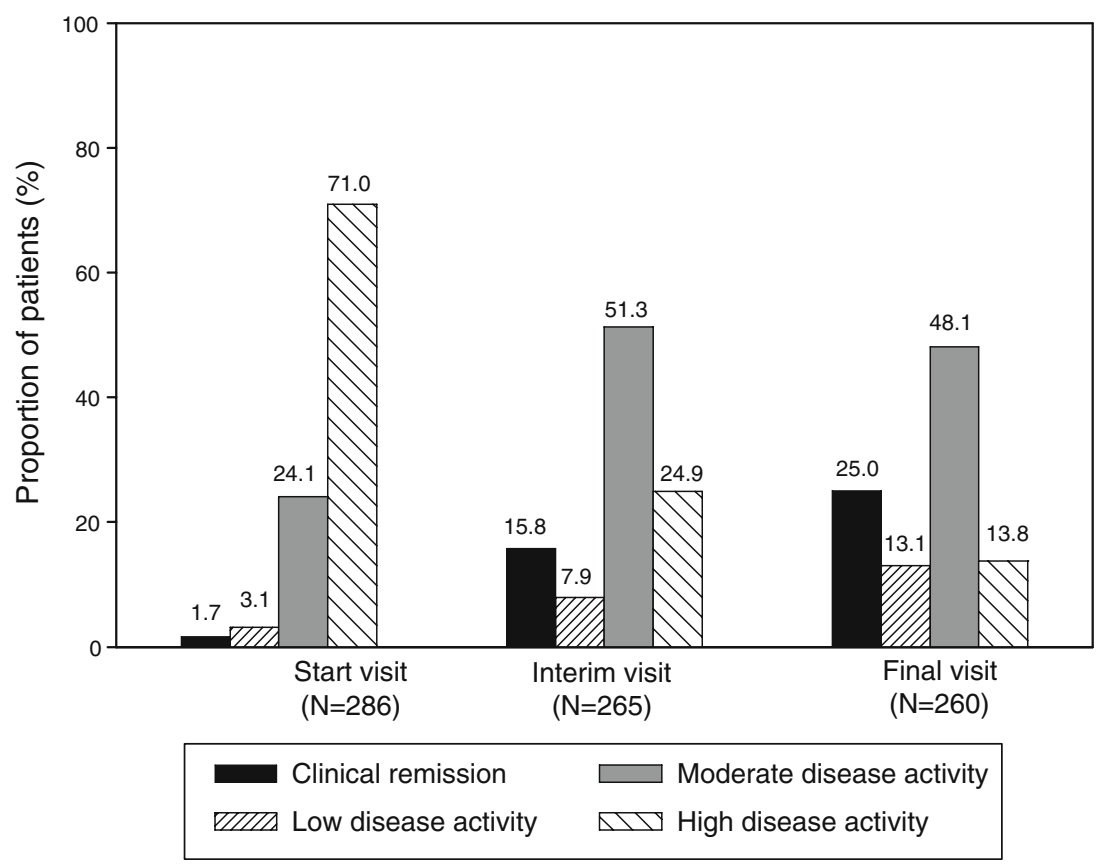

Neither for vital signs nor for body weight was relevant changes observed under treatment.

\section{Discussion}

RA patients with disease duration $\leq 1$ year since diagnosis treated by rheumatologists well distributed all over Germany were included in this non-interventional study, 16 months had passed between the occurrence of first symptoms and enrollment, thus the results can be considered as being representative for early RA patients under specialist care. Also with regard to age and gender, the patients represented a typical RA population with a mean age of 56 years and the majority of patients being women.

Analysis of the time between first RA symptoms and diagnosis shows that a considerable period can elapse before a final diagnosis is established. Whether this delay is caused by a long referral time from the general practitioner to the specialist can only be assumed. Given the importance of early and effective treatment of RA, optimizing the time to diagnosis seems necessary.

With respect to the inflammatory markers CRP and ESR and the measures of disease activity DAS28, TJC, and SJC, disease characteristics at baseline were overall comparable to those described for other early RA study populations [11,
Fig. 3 Course of DAS28 score and HAQ-DI score (effectiveness population). Whiskers represent standard deviation. $D A S$ disease activity score

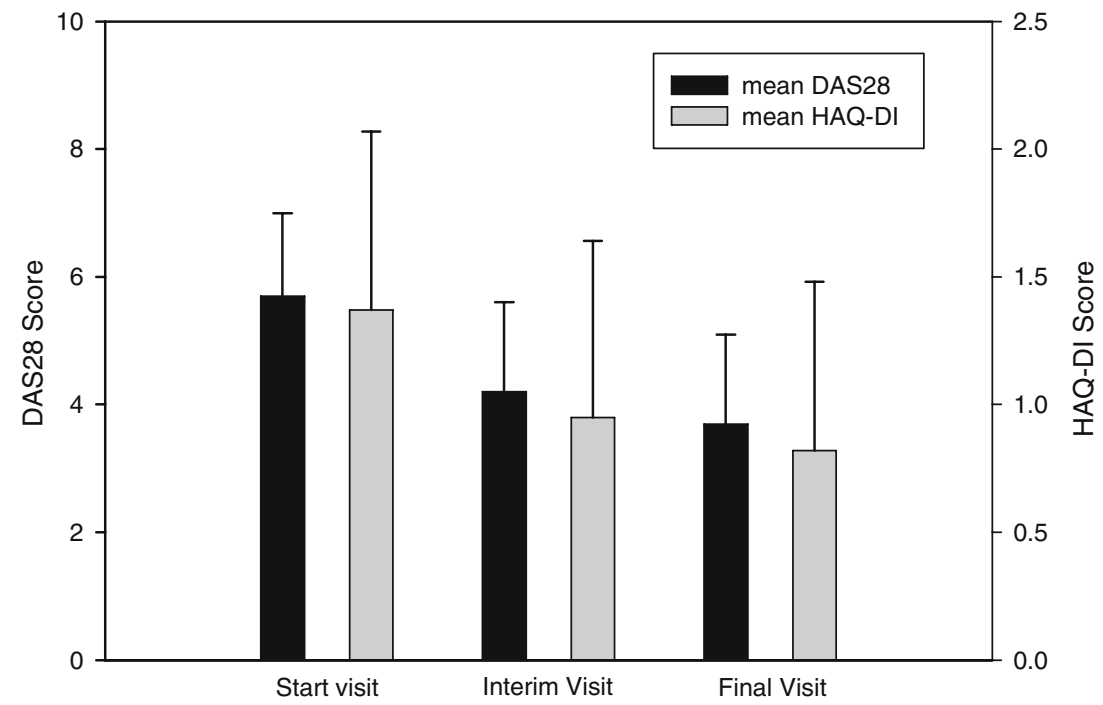


Table 3 Course of tender joint count, swollen joint count, erythrocyte sedimentation rate, and C-reactive protein (effectiveness population)

\begin{tabular}{lrcrrr}
\hline & TJC $($ mean \pm SD) & SJC $($ mean \pm SD) & ESR (mm/h, mean \pm SD) & CRP (mg/L, mean \pm SD) \\
\hline Baseline & $10.1 \pm 6.6$ & $8.1 \pm 5.7$ & $39.7 \pm 22.4$ & $37.7 \pm 80.5$ \\
Week 12 & $5.7 \pm 5.4$ & $4.1 \pm 4.2$ & $26.1 \pm 19.4$ & Not done \\
Week 24 & $3.9 \pm 4.7$ & $2.6 \pm 3.6$ & $23.2 \pm 17.2$ & $13.2 \pm 22.4$ \\
Difference week24-baseline $^{\mathrm{a}}$ & $-6.6 \pm 6.6$ & $-5.8 \pm 5.6$ & $-17.7 \pm 23.1$ & $-26.8 \pm 70.6$ \\
$P$ value for changes & $<0.001$ & $<0.001$ & $<0.001$ & $<0.001$ \\
\hline
\end{tabular}

CRP C-reactive protein, ESR erythrocyte sedimentation rate, $S D$ standard deviation, $S J C$ swollen joint count, TJC tender joint count

${ }^{a}$ Endpoint analysis, i.e., only patients considered with data both for baseline and week 24

${ }^{\mathrm{b}}$ Wilcoxon signed rank test

12]. However, baseline characteristics reflect the defined inclusion and exclusion criteria and these are often stricter in clinical studies where, in contrast to our noninterventional study, joint erosions or specific minimum tender or swollen joint counts may be required and patients might therefore present with a more severe illness.

Despite the relatively short disease duration in our study, joint erosions were already present in $45.6 \%$ of patients, again indicating the early occurrence of joint damage in the course of the disease.

As shown by the high DAS2 8 response rate, patients with early RA clearly profited from leflunomide treatment. Corresponding to the rapid onset of action of leflunomide described in the pivotal phase III study (US301) [6] where a positive effect regarding ACR20 response was seen after 1 month, a considerable DAS28 response rate of $71.9 \%$ was already seen at the interim visit after 12 weeks which further increased to $84.6 \%$ after 24 weeks. The high DAS28 response rate in our study at least matches the good response rates reported previously for leflunomide; in fact it is even higher. Dougados et al. found $69.6 \%$ of DAS28 responders after 24 weeks of treatment in the open phase of the RELIEF study [13]. However, disease characteristics in RELIEF at baseline with a longer disease duration (7 years) and a higher disease activity (mean DAS28: 6.3; high disease activity in $86.5 \%$ ) were markedly different from those in our study. Furthermore, a clearly higher proportion of patients had received DMARD pre-treatment. A further open-label study by Nguyen et al. [14] (disease duration 9.7 years; high disease activity at baseline in 63.0\%) reported $61.8 \%$ of DAS28 responders at 6 months. The short disease duration together with the fact that approximately $50 \%$ of the patients were DMARD-naïve could have added to the higher response rates observed in our study. However, DMARD pre-treatment on its own had no effect on the overall response rate although more "good responders" were seen in DMARD-naïve patients. The fact that anti-CCP which is known to predict an aggressive disease course with an unfavorable outcome [15] was only found in $60.9 \%$ of the patients ( $N=235$ patients with data available) might also have contributed. However, a subgroup analysis revealed no differences in DAS28-response in anti-CCP positive and negative patients.

A further subgroup analysis evaluating a possible influence of the administration of the loading dose on DAS28-response resulted in similar response rates in patients who had received a loading dose and those who had not. This finding adds new elements to the ongoing

Table 4 Course of HAQ-DI and pain (effectiveness population)

\begin{tabular}{|c|c|c|}
\hline & $\begin{array}{l}\text { HAQ-DI } \\
\text { (range 0-3) } \\
\text { Mean } \pm \text { SD }\end{array}$ & $\begin{array}{l}\text { HAQ-pain scale }(\mathrm{mm}) \\
(\text { range } 0-100) \\
\text { Mean } \pm \text { SD }\end{array}$ \\
\hline Baseline & $1.37 \pm 0.7$ & $60.3 \pm 21.2$ \\
\hline Week 12 & $0.95 \pm 0.69$ & $39.4 \pm 21.4$ \\
\hline Week 24 & $0.82 \pm 0.66$ & $29.0 \pm 20.0$ \\
\hline Difference week 24-baseline ${ }^{\mathrm{a}}$ & $-0.56 \pm 0.62$ & $-31.6 \pm 27.3$ \\
\hline$P$ value for changes ${ }^{\mathrm{b}}$ & $<0.001$ & $<0.001$ \\
\hline
\end{tabular}

$H A Q-D I$ health assessment questionnaire-disability index, $S D$ standard deviation

${ }^{a}$ Endpoint analysis, i.e., only patients considered with data both for baseline and week 24

${ }^{\mathrm{b}}$ Wilcoxon signed rank test

${ }^{\mathrm{c}}$ Current pain on day of assessment in contrast to average pain over last 7 days as in original HAQ 
discussion about the benefit/risk of the loading dose regimen for leflunomide, but since this is coming from a subgroup analysis from an observational study a conclusive answer is not possible and requires further clinical study.

The fact that concomitant corticosteroids and MTX had no influence on the DAS28-response reflects the naturalistic setting of this study and can be attributed to the patient channeling which takes place in daily clinical practice.

Corresponding to the high response rate all of the further parameters analyzed in this study showed great improvement under treatment. Clinical remission, defined as DAS2 $8 \leq 2.6$, was reached in $25 \%$ of patients. A similar remission rate $(24.4 \%)$ was seen in an open-label study with leflunomide in DMARD-naïve patients with early RA [16]. The remission rate in our study furthermore corresponds to the rates observed for anti-TNF-alpha and MTX monotherapy: In the PREMIER study with early RA patients who, however, presented with a more severe disease (higher values for SJC, TJC, HAQ-DI, and mean DAS28) DAS28 remission rates at year 1 were $23.0 \%$ for adalimumab and $21.0 \%$ for MTX [17]. The remission rate for MTX monotherapy in the COMET study was $28 \%$ at week 52 [18].

Reductions in mean DAS28, SJC, TJC, pain, and ESR at 3 months were comparable to those of an observational study with leflunomide on RA patients with longer disease duration [19]. Compared to the results for the subgroup of DMARD-naïve patients with early RA after 6 months of leflunomide treatment in the phase III study MN301, improvement with regard to pain in our study was very similar, while reductions in SJC and TJC were smaller [20]. Results obtained for the HAQ-DI underlined the benefit the patients gained in physical function by leflunomide treatment. A mean reduction by 0.56 with a minimum difference of 0.22 considered as clinically important [21] showed that functional ability was meaningfully improved under treatment. A comparable outcome was observed in the US301 phase III study [6], where interestingly, with markedly higher swollen and tender joint counts, the HAQDI score at baseline was quite similar to the one in our study.

The results of the safety evaluation were consistent with the known safety profile of leflunomide and no new safety risks were observed.

\section{Conclusions}

Leflunomide showed the effectiveness which was found in controlled studies without revealing any new or hitherto unknown side effects. Onset of action was quick and significant improvement of disease was seen after 12 weeks of therapy and at even higher rates after 24 weeks.
Interestingly, the remission rate achieved was similar to the rate seen with methotrexate or biologic therapy in other studies. This outlines the importance of the potential benefit of leflunomide in the treatment of early RA patients in routine rheumatology setting.

Disclosures H.K. has received speakers' honoraria from SanofiAventis Deutschland GmbH, Germany.

K.B. is full-time employee of Sanofi-Aventis Deutschland GmbH, Germany.

G.H. has received speakers' honoraria from Sanofi-Aventis Deutschland GmbH, Germany.

This study was funded and managed by Sanofi-Aventis Deutschland GmbH Germany (LEFLU_L_01532).

Open Access This article is distributed under the terms of the Creative Commons Attribution Noncommercial License which permits any noncommercial use, distribution, and reproduction in any medium, provided the original author(s) and source are credited.

\section{References}

1. Lard LR, Visser H, Speyer I et al (2001) Early versus delayed treatment in patients with recent-onset rheumatoid arthritis: comparison of two cohorts who received different treatment strategies. Am J Med 111:446-51

2. Emery P, Salmon M (1995) Early rheumatoid arthritis: time to aim for remission? Ann Rheum Dis 54:944-7

3. Aletaha D, Eberl G, Nell VP et al (2004) Attitudes to early rheumatoid arthritis: changing patterns. Results of a survey. Ann Rheum Dis 63:1269-75

4. Machold KP, Stamm TA, Eberl GJ et al (2002) Very recent onset arthritis - clinical, laboratory, and radiological findings during the first year of disease. J Rheumatol 29:2278-87

5. Sharp JT, Strand V, Leung H et al (2000) Treatment with leflunomide slows radiographic progression of rheumatoid arthritis: results from three randomized controlled trials of leflunomide in patients with active rheumatoid arthritis. Leflunomide Rheumatoid Arthritis Investigators Group. Arthritis Rheum 43:495-505

6. Strand V, Cohen S, Schiff M et al (1999) Treatment of active rheumatoid arthritis with leflunomide compared with placebo and methotrexate. Leflunomide Rheumatoid Arthritis Investigators Group. Arch Intern Med 159:2542-50

7. Dayer J-M, Cutolo M (2005) Is there a rational to using leflunomide in early rheumatoid arthritis? Clin Exp Rheumatol 23:404-12

8. Smolen J, Emery P, Kalden J et al (2004) The efficacy of leflunomide monotherapy in rheumatoid arthritis: towards the goals of disease modifying antirheumatic drug therapy. J Rheumatol 71:13-20

9. van Gestel AM, Prevoo ML, van 't Hof MA et al (1996) Development and validation of the European League Against Rheumatism response criteria for rheumatoid arthritis. Comparison with the preliminary American College of Rheumatology and the World Health Organization/International League Against Rheumatism Criteria. Arthritis Rheum 39:34-40

10. Bruce B, Fries JF (2003) The Stanford health assessment questionnaire: a review of its history, issues, progress, and documentation. J Rheumatol 30:167-78 
11. Sanmarti R, Gomez A, Ercilla G et al (2003) Radiological progression in early rheumatoid arthritis after DMARDS: a oneyear follow-up study in a clinical setting. Rheumatology (Oxford) 42:1044-9

12. Jansen L, van der Horst-Bruinsma IE, van Schaardenburg D et al (2001) Predictors of radiographic joint damage in patients with early rheumatoid arthritis. Ann Rheum Dis 60:924-7

13. Dougados M, Emery P, Lemmel E et al (2003) Efficacy and safety of leflunomide and predisposing factors for treatment response in patients with active rheumatoid arthritis: RELIEF 6-month data. J Rheumatol 30:2572-9

14. Nguyen M, Kabir M, Ravaud P (2004) Short-term efficacy and safety of leflunomide in the treatment of active rheumatoid arthritis in everyday clinical use. Clin Drug Investig 24:10312

15. Skogh T (2005) Does a positive anti-CCP test identify a distinct arthritis entity? Arthritis Res Ther 7:230-2

16. Alperi-Lopez M, Ballina-Garcia F, Riestra-Noriega J et al (2005) Leflunomide treatment in patients with early rheumatoid arthritis and non prior DMARD therapy. Ann Rheum Dis 64(Suppl III): 468
17. Breedveld F, Weisman M, Kavanaugh A et al (2006) The PREMIER study: a multicenter, randomized, double-blind clinical trial of combination therapy with adalimumab plus methotrexate versus methotrexate alone or adalimumab alone in patients with early, aggressive rheumatoid arthritis who had not had previous methotrexate treatment. Arthritis Rheum 54:26-37

18. Emery P, Breedveld FC, Hall S et al (2008) Comparison of methotrexate monotherapy with a combination of methotrexate and etanercept in active, early, moderate to severe rheumatoid arthritis (COMET): a randomised, double-blind, parallel treatment trial. Lancet 372:375-382, Epub 2008 Jul 16

19. Wendler J, Schuch F, de la Camp R (2003) Efficacy and tolerability of leflunomide in treating rheumatoid arthritis (RA) under practice conditions: results of an observational study of usage in 1420 patients. Ann Rheum Dis 62(Suppl 1):180

20. Smolen J, Breedveld F, Strand V et al (2002) Efficacy of leflunomide in DMARD-naïve patients with early RA. Ann Rheum Dis 61(Suppl I):166

21. Strand V (2004) Longer term benefits of treating rheumatoid arthritis: assessment of radiographic damage and physical function in clinical trials. Clin Exp Rheumatol 22:S57-64 